\title{
Interface Management during Transportation of Products through Multi-Product Petroleum Pipelines without Kerosene Plug
}

\author{
Mohd Sadaf ${ }^{1}$ (B)
}

Received: 16 August 2017/ Accepted: 12 July 2018/Published online: 19 July 2018

(C) The Institution of Engineers (India) 2018

\begin{abstract}
This is an innovative work that may prove to be a milestone in terms of saving money that gets wasted due to the generation of unwanted interface $(\mathrm{I} / \mathrm{F})$ mixture between different petroleum products (diesel, gasoline and kerosene oil) in direct contact, that are being pushed backto-back through the same pipeline as per current industrial practice. It is known that the interface between any two liquids is generated due to diffusion as per Fick's first law of diffusion. Industrial diesel is often called high speed diesel (HSD), gasoline is called motor spirit (MS) and kerosene oil is called superior kerosene oil (SKO). Now, let's take the example of pumping of HSD-SKO-MS, in that order, through the pipeline. It is obvious that the amount of diffusion, hence, interface generation, increases with the increase in the time for which they are in contact. That is why, if its' considered to be shutdown, the interface quantity increases. The presence of HSD in MS adversely affects its Octane no. and other basic properties. So, HSD is not desirable to be allowed to get mixed with MS batch. The present study is discussed about how to manage the interface without SKO plug so that the product quality is meeting the Industry standards. In this work, it has been proposed for using ethanol (aqueous) as a plug between MS and HSD, which are being pushed back-to-back through the pipeline as per current industrial practice.
\end{abstract}

Keywords Petroleum - Interface management . Superior kerosene oil (SKO) · Petroleum pipelines

Mohd Sadaf

mohdsadaf1985@gmail.com; mohammed@indianoil.in

1 Indian Oil Corporation Limited (Pipelines), Allahabad, India

\section{Notations}

${ }^{\circ} \mathrm{C} \quad$ Degree centigrade

BKPL Barauni-Kanpur pipeline (for petroleum products)

BSIII Bharat Standard III (emission)

BSIV Bharat Standard IV (emission)

HSD High speed diesel

I/F Interface, that is, the mixture of the two petroleum products that are pushed back to back through the same pipeline

kl Kilolitres

MS Motor spirit (gasoline)

SKO Superior kerosene oil

$\mathrm{v} / \mathrm{v} \quad$ Volume/volume ratio

\section{Introduction}

This is an innovative work that may prove to be a milestone in terms of saving money that gets wasted due to the generation of unwanted Interface (I/F) between different petroleum products that are being pushed back to back through the same pipeline as per current industry practice. It is well known that the interface between any two liquids (A and $\mathrm{B}$ ) is generated due to diffusion that is dependent on:

1. Chemical potential gradient;

2. Difference in concentration of liquid $\mathrm{A}$ in $\mathrm{A}$ and $\mathrm{B}$ and that of liquid B in A and B as per Fick's first law of diffusion.

Industrial diesel is often called high speed diesel (HSD), gasoline is called motor spirit (MS) and kerosene oil is called superior kerosene oil (SKO). Now, let's take the 
example of pumping of HSD-SKO-MS, in that order, through the pipeline. It is obvious that the amount of diffusion and hence the interface generation, increases with the increase in the time for which they are in contact. So in case of shutdown, the interface quantity increases.

Also, to sustain turbulent flow, Reynold's number is generally in the range of 60,000 to 110,000 or so for BKPL, so as to avoid laminar flows or flows through layers.

\section{The Challenge}

Currently, SKO has been used as a plug between MS and HSD batched flowing back to back in the same pipeline in the order MS-SKO-HSD-SKO-MS... at high pressure (in the range of 30 to 75 bars) and Reynolds number around 100,000 (turbulent). This causes interface between MSSKO and SKO-HSD that has to be absorbed in MS and HSD tanks respectively and SKO is kept pure (as per statutory guidelines).

But, with the growing demand of natural gas and decreasing requirement of SKO, soon SKO pumping through pipeline would have to be stopped. When HSD and MS would be pushed back to back without any suitable plug through the pipeline, huge quantity of interface would be generated that would destroy their specifications. The presence of HSD in MS adversely affects its Octane no. and other basic properties. So, HSD is not desirable to be allowed to get mixed with MS batch.

The methodology to manage the interface without SKO plugs to meet the product quality as per industry standards is discussed in the study.

\section{Using Ethanol as a Plug between MS and HSD}

The basis of the proposal is as follows:

- Under most conditions ethanol readily blends with gasoline at all ratios. unlike gasoline, diesel fuel is not easily blended with ethanol;

- Aqueous ethanol (content $99.6 \% \mathrm{v} / \mathrm{v}$ at $15.6{ }^{\circ} \mathrm{C}$, as per IS 15464:2004) blended MS is already being sold (10\% $\mathrm{v} / \mathrm{v})$ by marketing.

- Ethanol-MS or MS-ethanol interface may be absorbed in MS tanks at, say, up to 5\% v/v. Rest of the blending could be done at terminal to meet the requirement of ethanol (10\% v/v) as per Industry Quality Control Manual.

Density of ethanol (maximum $795.6 \mathrm{~kg} / \mathrm{cu}-\mathrm{m}$ at $15.6{ }^{\circ} \mathrm{C}$, as per IS $15464: 2004$ ) is near to that of SKO, hence interface tracking/cutting would be easy. Because interface tracking/cutting for SKO has also been carried out similarly, that is, on density monitoring basis.

\section{Current procedure using SKO as a plug between MS and HSD}

Currently, SKO has been used as a plug between MS and HSD batched flowing back to back in the same pipeline in the order MS-SKO-HSD-SKO-MS...at high pressure (in the range of 30 to 75 bars) and Reynolds number around 100,000 (turbulent). This causes interface-generation between MS-SKO and SKO-HSD that has to be absorbed in MS and HSD tanks, respectively and SKO is kept pure (as per statutory guidelines). Thus, the Refinery has to push these two products with better-than-required quality so that at the user end, after mixing of interface, the final product meets BSIV norms. This loss is huge in terms of revenue.

In the storage-cum-pumping station at Barauni, near the crude oil Refinery unit, different petroleum products are stored in designated above ground storage tanks. Petroleum products (MS, HSD, and SKO) are pushed from the mother station through the pump station there, that boosts up the pressure and generates the flow through the pipeline going from Barauni to downstream delivery/terminal stations like Allahabad, where the products are received in respective tanks. The petroleum products are received at the delivery stations like Allahabad with the help of operating personnel who operate valves to start/stop/changeover the product's delivery in the respective tanks (MS, SKO or HSD). As the different products are pushed back to back through the same pipeline, mixing takes place. To avoid mixing of MS and HSD, calculated quantity of SKO is pushed in between MS and HSD in the sequence: MS-SKO-HSD-SKO-MSSKO-HSD... and so on.

The density of product flowing through the pipeline is monitored in real-time and $\mathrm{I} / \mathrm{F}$ tracking/cutting is done on the basis of density of the product. All the petroleum products like MS, SKO and HSD have considerably variable density range. This helps to trace which product is flowing through the delivery station pipeline at any moment, whether it is pure product (density value same as that for respective product tank at mother station) or an interface of any two products ( $\mathrm{I} / \mathrm{F}$ has a density value in between those two products being pushed back to back). For example, delivery of incoming pure SKO is going on in an SKO tank at the delivery/terminal station. As soon as the density of flow changes from that pure SKO, it is known that the I/F has reached the delivery station. Then the change over/diversion of the flow is occurred into the respective storage tank. When the density reaches the value of pure MS and stabilizes, it reveals that the I/F has passed through. The MS-SKO interface has to be absorbed in MS 
at $2 \%$ by volume as per Industry Quality Control Manual (IQCM) while SKO has to be kept pure.

Similarly, SKO-HSD interface has to be absorbed in HSD at $3 \%$ by volume. But, with the decreased use of SKO, soon the demand of SKO to be delivered through pipeline is stopped. Then, without SKO plug, MS and HSD would mix as undesirable manner since that would destroy the required specifications of MS and HSD as per IQCM. A solution to this grave problem is suggested by pushing aqueous ethanol as a plug between MS and HSD.

\section{Proposed Procedure using Aqueous Ethanol (96 to $99.6 \% \mathrm{v} / \mathrm{v}$ ) as a Plug between MS and HSD}

MS batch shall be pushed through the pumps fitted for pushing the petroleum products through pipeline from MS tanks at the pump station near refinery. Pumping changeover would be taken at the mother storage-cum-pumping station (Barauni) itself, from HSD to ethanol (just like it is obtained for SKO as per current practice) and it would be pushed as per pre-decided quantity (4 to 5\% of MS batch). Then again change-over shall be taken, this time to MS tank as per practice. The only change in the process is changing over the pumped product in the order HSDethanol-MS instead of current practice, that is, HSD-SKOMS.

Ethanol may be supplied and filled into a dedicated ethanol tank located in the pipeline division's tank farm. Ethanol tank dip before and after the pushing of ethanol Plug would have to be measured jointly with marketing division to gauge the amount of ethanol pushed.

\section{Expected Interface Profile}

MS-ethanol interface would form primarily due to diffusion as their densities are different and diffusivity of ethanol in MS is high. The MS-ethanol interface would be followed by pure ethanol. Then a small ethanol-HSD interface is expected as ethanol is almost immiscible with HSD and hence diffusion should be negligible. 'Under most conditions ethanol readily blends with gasoline at all ratios. Unlike gasoline, diesel fuel is not easily blended with ethanol under all conditions' [1]. Manual mixing should also be minimum, due to the absence of Laminar flow in the pipeline. This would be followed by pure HSD batch as shown in Fig. 1.

\section{Interface Management}

MS-ethanol I/F along with pure ethanol would be absorbed in MS, though it will not deteriorate the specifications of

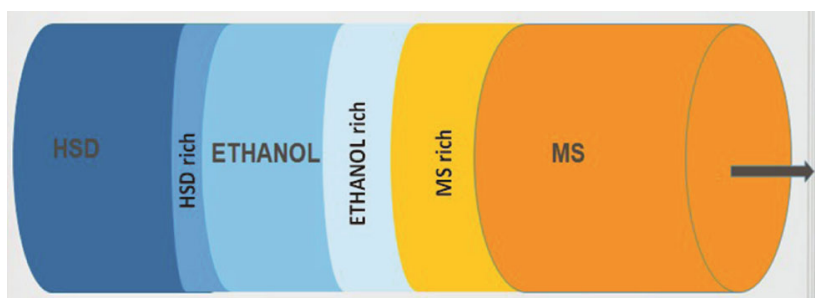

Fig. 1 Petroleum product profile inside the pipeline. Note The four segments between HSD and MS in this figure. That is the expected I/F profile

MS, since MS-ethanol blend (10\% v/v) is already being retailed in the market. Rather, ethanol is an Octane booster.

$\mathrm{I} / \mathrm{F}$ tracking would be very easy since the density of ethanol (specific gravity $=0.7956$ and concentration $=99.6 \% \mathrm{v} / \mathrm{v}$ at $15.6{ }^{\circ} \mathrm{C}$, as per IS 15464:2004) is around that of SKO. That means no additional skill is required for $\mathrm{I} / \mathrm{F}$ tracking/cutting. For BKPL, the minimum batch length of MS is $7000 \mathrm{kl}$. So, ethanol batch required at $5 \%$ would be $350 \mathrm{kl}$ as per Operation manual of BKPL. Thus a plug of say $175 \mathrm{kl}$ may be put at both the front and rear end of MS batch. As per a sample data (MS-SKO I/F (Barauni-generated) received at BKPL, Allahabad on December 06, 2016), the I/F quantity was $123 \mathrm{kl}$ while that of SKO-HSD received on December 07, 2016 was $81 \mathrm{kl}$ only.

Ethanol is miscible with MS just like SKO and its density also is almost same. So a well interface can be drawn that the I/F quantity of MS-ethanol won't exceed $175 \mathrm{kl}$ (ethanol batch-length at 5\% of MS batch). Although, the presence of ethanol in HSD causes a decrease in its viscosity and heat content, but, 'with an ethanol content of 10 to $20 \%$, the viscosity does not reach the minimum requirement for diesel fuels' [1].

The ethanol-HSD I/F (theoretically negligible) would have to be absorbed in HSD tank HSD is desirable in MS since it decreases its Octane number. This relatively small amount of ethanol in HSD can be got absorbed by adding some amount of bio-diesel in the HSD tank [2]. The work on exploring the amount of bio-diesel that can be mixed with HSD has been going on and by the time the proposal of complete removal of SKO plug may be implemented. Further, these must have facilities for bio-diesel blending with HSD, so that the said constraint would be eliminated easily.

Ethanol doesn't have oxidizing properties and its $\mathrm{pH}$ is neutral and is not corrosive to metals. That is why it has been transported through mild steel, stainless steel containers and is also being used in automobile engines. Also, corrosion inhibitors are being used in pipelines for petroleum products like MS and HSD already. Similarly, a suitable corrosion inhibitor would be used for ethanol as well that shall take care of the minimal corrosion that it 
could cause. Further, transportation of ethanol through pipelines has already started at some countries. Most of the petroleum pipelines in India and other countries have outlived their designed life, new pipelines are being laid. So the new pipeline material may be selected accordingly to ensure minimum corrosion with ethanol.

\section{Conclusion}

Ethanol can replace SKO as a plug between MS and HSD (that are highly miscible) because "under most conditions Ethanol readily blends with gasoline (MS) at all ratios. Unlike gasoline, diesel fuel (HSD) is not easily blended with ethanol under all conditions'. Thus, the small quantity of HSD-ethanol interface generated, can be absorbed in HSD by adding some amount of bio-diesel in the HSD tank; while whole of ethanol-MS interface may be absorbed in MS as MS-ethanol blend of $10 \% \mathrm{v} / \mathrm{v}$ is already being retailed in the market. So, the product downgrading would be completely eliminated that shall save millions to the oil sector. This is due to the density of ethanol ( $0.7956 \mathrm{~g} / \mathrm{cum}$ at $15.6{ }^{\circ} \mathrm{C}$, as per IS $\left.15464: 2004\right)$ being very close to that of SKO and no additional skill is required for density-monitoring based interface tracking/cutting that is prevalent in the industry for SKO-HSD and SKO-MS interface.

\section{References}

1. D.G. Li, H. Zhen, L. Xingcai, Z. Wu-Gao, Y. Jian-Guang, Renew. Energy 30, 967-976 (2005)

2. M. Al-Hassan, Jordan J. Mech. Ind. Eng. 6, 147-153 (2012) 